\title{
Working Rural Eco Tourism Planning in Yogyakarta Using MSP+DM Analysis
}

\author{
Setiawan Priatmoko
}

STIE Pariwisata API Yogyakarta

Corresponding Author : eraynesance@gmail.com

\begin{abstract}
ARTICLE INFO ABSTRACT
Received

15 December 2017

Ecotourism planning requires a systematic simple tool. This is because Accepted

13 March 2018

Available online

30 March 2018 generally the actors of ecotourism in the region of Yogyakarta is a rural community activist which need a simple tools. Ecotourism activities are often associated with village tourism activities managed by the local community as community based tourism (CBT). Using MSP + DM analysis helps facilitate the planning and development of ecotourism for all stakeholders.
\end{abstract}

Keywords: ecotourism, planning, rural, CBT, MSP+DM

\section{Introduction}

\section{Background}

Ecotourism has become an important part in rural tourism activities in Yogyakarta. Most of the tourist villages make activities in the natural landscape as part of the attraction. Euphoria occurs in the community in response to the massive of tourism activities and the potential of nature that is considered to be developed as a tourist destination. Encouraging the massive use of eco-tourism issues become important. The countryside in the special region of Yogyakarta generally has a unique natural condition. Starting from the mountains, hills, plains, and coastal areas. In general, these different natural conditions become part of comparative advantage when compared to other areas and also for prospective visitors from other regions. Utilization of nature for this tourism aspects still largely managed and initiated by the local community. Starting from the determination, design, and operational activities of eco tourism usualy done by the community and sometimes by local government. The role of government is to facilitate infrastructure and also to withdraw retribution or other schemes.

http://ojs.unud.ac.id/index.php/eot
Rural in the suburbs of Yogyakarta often combine cultural activities and activities in the wild as attractions to attract tourists. The emergence of social media often feature landscapes or activities in the open space activities encourages community groups in villages to explore the potential of nature in the region to be sold as a tourist destination. Tourism stakeholder initiatives in developing tourist spots generally use trial and error systems, follow other areas that are considered successful, or use SWOT analysis. SWOT analysis result generate different management strategies among village stakeholders. The diferent of scientific background level and understanding about the uniqueness of tourism businesses among stakeholders also influences how the strategy of managing the eco-tourism assets. In such cases will be found some villages that are considered successful in selling eco-tourism activities but elsewhere there are areas that are considered failed to bring visitors. Not only the number of tourist visits is sometimes become a question for the stakeholders. The issue of sustainability has also been an issue that poses challenges to ecotourism activities initiated by the community. In addition, the local community's participation in ecotourism business activities in his village is also raising question for some 
other residents. Profit sharing and transparency are still a big issues that few villagers dare to ask or even to know how to measure an activity that is considered participatory for all villagers. Another thing that is important in Yogyakarta is the low awareness of citizens and stakeholders that eco tourism business should also pay attention to disaster mitigation. In general the villagers life style has not considered disaster mitigation as one of the important variables of an ecotourism activity. The conditions of such problems require a solution in the form of a relatively easy to use, comprehensive, and consistent tool to design of an eco-tourism area. One such analytical tool is the analysis of MSP+DM, which measures the aspects of Marketing (Marketibility), Sustainability, Participatory, and Disaster Mitigation Management.

\section{Research Objectives}

Based on the explanation of the problems above, this paper will discuss about the MSP+DM analysis as an analysis tool to determine, designing processes, and setting goals of ecotourism business in Yogyakarta. This study focuses on eco-tourism activities conducted by rural communities as community-based tourism (CBT). Ecotourism activities are viewed as a community-based tourism activity. Different conditions of understanding on society about ecotourism business require a simple guide to the direction of planning and development. This should be really useful and well targeted. The MSP + DM analysis will be tested whether it is truly appropriate as one of the tools to develop an ecotourism area. Objectives of the research are:

a. to review the variables of ecotourism activities based on community through literature study

b. to learn the condition of eco tourism activities in Yogyakarta

c. to examines the variables of ecotourism activity on the MSP + DM analysis tool

\section{Literature Review}

Geographically, the Special Province of Yogyakarta has a land area of 318.58 hectares and $2169.6 \mathrm{~km} 2$ ocean $(12$ miles ocean) (BAPPEDA, 2009). These areas of

http://ojs.unud.ac.id/index.php/eot land use is in the form of various forms. Various forms and utilization of this land that will be one factor to be used as ecotourism. The great number of land in Yogyakarta is forest area followed by rice field and settlement. Utilization of forests and rice fields for ecotourism activities in Yogyakarta is generally done by rural community groups. Such community groups are commonly affiliated in tourism village entities. Ecotourism is responsible travel to natural areas that conserves the environment and sustains the well being of local people (Wood, 2002). The UNWTO's definition, ecotourism refers to forms of tourism which have the following characteristics, such as (i) All nature-based forms of tourism, observation and appreciation is the main motivation, (ii) The tourism activities contains educational and interpretation features. It is generally, but not exclusively organised by specialised tour operators for small groups, (iii) The bussiness organizations tend to be small and owned by local people, (iv) Tend to minimizes negative impacts upon the natural and socio-cultural environment, and (v) It supports ecotourism attractions natural areas by: Generating economic benefits for local communities, organisations and stakeholders managing natural areas with conservation purposes; Providing alternative employment and income ads for local communities; Increasing awareness towards the conservation of natural and cultural assets, both among locals people and visitors.

The consequences of natural-based utilization and responsible utilization require serious attention prior to development as a tourist area. Ecotourism usually using protected areas that strives to be low impact and (often) small scale (Sayyed, 2013). But in its development process, ecotourism, nature, heritage, cultural, and soft adventure (e.g.: fish catching, bird watching, and milking) tourism are taking the lead and are predicted to grow rapidly over the next two decades. It is estimated that global spending on ecotourism is increasing about six times the industry-wide rate of growth (UNEP, 2011). For the protection of natural assets and side effects and the utilization of eco-tourism activities need to be carefully taken into account. As an activity that has a business purpose, ecotourism is related to marketing activities. 
The marketing aspects involved in ecotourism activities by Priatmoko (Ayub et al., 2013) are: (i) Determination of the tourism market. (ii) Products that uniqueness and originality typical of the local area which is regarded as a tourist attraction, (iii) Price is an obtained agreement on the profit margin, and its distribution among tourism businesses, (iv) Distribution is the stability of the availability of various tourism products and can be accesed by consumers/travelers, and (v) Promotion in term of communication dissemination of information on the local tourism product range.

Ecotourism is a special interest activity tourism. Every area will have special uniqueness and originality, the prices are also setting up differently with another mass tourism bussines. The role of marketing strategy is needed for various purposes i.e. gaining competitive advantage, counteracting markets' insurgencies, and identifying market potentials (Ayub et al., 2013). Strategy of marketing also need differentiated approach: Provides marketing mix in a different way than competitive destinations Oreski (2012). This will be definetely including kind of product, price, place, promotion, people/ market. Marketing efforts can be focused by developing the most effective message for the segment targeted. In Yogyakarta ecoturism activities the example is bird watching for animal lover or fish catching for river activities lover. Furthermore communicating the message through the most effective communication channel for the segment is also important (Dolnicar, 2008), i.e., using Instagram or other social media platform for student visitors). There is no doubt that communication through social media will affect visits to destinations (Priatmoko, 2017b).

Analysis MSP + DM requires sustainability variables to be assessed. The aspects of sustainibilty include the concern of (i) carrying capacity of tourism products, (ii) waste treatment, (iii) preservation of the environment, (iv) the continuity of tourist visit, and (v) business development actors and local related groups (Priatmoko, 2017a). Ecotourism is a sub-component of the field of sustainable tourism. Sustainable meanings are also not limited to the sustainability of

http://ojs.unud.ac.id/index.php/eot nature but also include in the face of changing economic, social and cultural patterns and widespread rural restructuring (Dashper, 2015). Sustainable issue in tourism not only the expectations of tourists regarding responsible natural- resource management (demand), but also the needs of communities that support or are affected by tourism projects (in this case is ecourism project) and the environment (supply) (UNEP, 2011). From the sustainable concept it shows that the sustainability of the continuity of visit and business development must be considered. In the development of ecotourism area the role of local community participation is became important. Promoting sustainable value chains within local communities need to be done. The need is to ensure a consistent quality and creativity, fostering local training and educational opportunities in creative and cultural industries; and encouraging public and private investment to sustain local creative industries among local people (UNWTO, 2016).

Participatory variabel also be assessed in MSP+DM analysis. Participatory values in MSP+DM regard to the following matters, (i) Main role by local communities , (ii) Responsible for local accountability, (iii) Focus in Local variety. Sustainable tourism development, in particular of local communities, need to be ensured to the wide participation (UNWTO, 2011). One of most definitions of ecotourism need to meets meaningful for community participation (Dorobantu and Nistoreanu, 2012). Public participation in tourism activity like ecotourism can take many forms, including organizing tourism advisory committees or boards (Spencer, 2010).

Generaly ecotourism management in Yogyakarta is conduct by villagers. Usualy their understanding of disaster mitigation is still not similar and standard. Safety still needs to be reinforced among the managers and stakeholders. UNWTO also promote and ensure the need global awareness to future hazards of any kind to improve regional and global efforts (UNWTO, 2011). Disaster consists of two kinds: natural disaster and nonnatural disaster. Natural disaster is is an event or series of events that threaten and disrupt the lives and livelihoods caused by natural factors 
(e.g.: earthquake, tsunami, volcanic eruption) that can lead to result in loss of material and life (BPS, 2017). For Yogyakarta community the understanding on disaster characteristic is a warranty of safety investment in the future (Susmayadi et al., 2014). During 2015-2016 Yogyakarta had 12 life fatality, 11 injury, and 1 person missin. Using MSP+DM analysis disaster mitigation can be focused in: (i) natural disasters (e.g.: earth quake, volcano eruption, tsunami, storm), and (ii) non-natural disasters, especially for handling the risks that may arise for the products and or human error (Priatmoko, 2017a). A rational approach to aware lifting aspects of Disaster Mitigation can be key the development of a tourist area in the long term (Purbadi, 2016).

\section{Methodology}

MSP+DM approaches which is a measurement method based on the aspects of Marketing (Marketibility/M), Sustainability (S), Participatory (P), and Disaster Mitigation (DM) Management was used to analyse the data information in order to determine, design processes, and set the goals of ecotourism business inYogyakarta. This study focuses on ecotourism activities which are viewed as community-based tourism (CBT) activity conducted by rural communities. Different conditions of understanding on society about ecotourism business require a simple guide to the direction of planning and development. The MSP + DM analysis will be tested whether it is truly appropriate as one of the tools to develop an ecotourism area.

MSP + DM was undertaken based on setting and selecting the attractions that already exist and the potential attractions as contents that will be analyzed and revealed in the future quantitatively. Because ecotourism is a product that contains aspects of marketibility, sustainibility, participatory, and need emphasis in disaster mitigation using the parameters of MSP+DM analysis tools very compatible. The use of scores based on the presence level of the indicators will also facilitate easily for stakeholders to establish of the basis planning area. The rank of the Value Scale \& Categories of a region's initial condition become embryo, pioneer, growing, and advance which will help stakeholders in determining the steps of development as can be seen in Table 1.

Table 1. Values Scale \& Categories

\begin{tabular}{cc}
\hline Scales & Categories \\
\hline $1.00-1.99$ & Embryo \\
$2.00-2.99$ & Pioneer \\
$3.00-3.99$ & Growing \\
$4.00-5.00$ & Advance \\
\hline
\end{tabular}

Source: Priatmoko, 2017a

\section{Results and Discussion}

Results of the MSP+DM analysis can be seen in Table 2 which show that stakeholders can determine more advance target using bigger points in MSP+DM. The criteria for improving the condition of the area exist in the content of evaluation parameters of MSP+DM.

Table 2. Content of evaluation parameters of MSP + DM Scale

\begin{tabular}{|c|c|c|c|c|c|c|}
\hline \multicolumn{7}{|c|}{ 1. MARKETIBILITY } \\
\hline \multirow{2}{*}{$\begin{array}{l}\text { PARA } \\
\text { METE } \\
\text { R }\end{array}$} & \multirow{2}{*}{$\begin{array}{l}\text { DET } \\
\text { AIL }\end{array}$} & \multicolumn{5}{|c|}{ VALUES \& INDICATORS } \\
\hline & & $\begin{array}{c}1 \\
\text { point }\end{array}$ & $\begin{array}{l}2 \\
\text { points }\end{array}$ & $\begin{array}{l}3 \\
\text { poin } \\
\text { ts }\end{array}$ & $\begin{array}{l}4 \\
\text { points }\end{array}$ & $\begin{array}{l}5 \\
\text { points }\end{array}$ \\
\hline $\begin{array}{l}\text { A. } \\
\text { Produc } \\
\mathrm{t} \\
\text { Packag } \\
\text { ing }\end{array}$ & $\begin{array}{l}\text { the } \\
\text { pack } \\
\text { agin } \\
\text { g of } \\
\text { good } \\
\text { s or } \\
\text { servi } \\
\text { ces } \\
\text { appe } \\
\text { aranc } \\
\text { e }\end{array}$ & $\begin{array}{l}\text { If there } \\
\text { is no } \\
\text { agreem } \\
\text { ent for } \\
\text { the } \\
\text { packag } \\
\text { ing of } \\
\text { various } \\
\text { types } \\
\text { of } \\
\text { tourism } \\
\text { product } \\
\text { s }\end{array}$ & $\begin{array}{l}\text { If using } \\
\text { standar } \\
\mathrm{d} \text { for } \\
\text { packag } \\
\text { ing } \\
\text { several } \\
\text { types } \\
\text { of } \\
\text { tourism } \\
\text { product } \\
\mathrm{s} \text {, but } \\
\text { not for } \\
\text { all its } \\
\text { product } \\
\mathrm{s} \text { yet }\end{array}$ & $\begin{array}{l}\text { If } \\
\text { using } \\
\text { prod } \\
\text { uct } \\
\text { stand } \\
\text { ardiz } \\
\text { ation } \\
\text {, } \\
\text { howe } \\
\text { ver, } \\
\text { for } \\
\text { certa } \\
\text { in } \\
\text { types } \\
\text { of } \\
\text { busin } \\
\text { ess } \\
\text { only }\end{array}$ & $\begin{array}{l}\text { If there } \\
\text { are } \\
\text { agreem } \\
\text { ent and } \\
\text { standar } \\
\text { dizatio } \\
\mathrm{n} \text { of } \\
\text { forms } \\
\text { and all } \\
\text { kinds } \\
\text { packag } \\
\text { ing of } \\
\text { tourism } \\
\text { product } \\
\mathrm{s}\end{array}$ & $\begin{array}{l}\text { If there } \\
\text { are } \\
\text { agreem } \\
\text { ent and } \\
\text { standar } \\
\text { dizatio } \\
\mathrm{n} \text { of } \\
\text { forms } \\
\text { and all } \\
\text { types } \\
\text { of } \\
\text { tourism } \\
\text { product } \\
\mathrm{s} \text { and } \\
\text { themati } \\
\mathrm{c} \\
\text { packag } \\
\text { ing } \\
\text { continu } \\
\text { ity }\end{array}$ \\
\hline $\begin{array}{l}\text { B. } \\
\text { Promot } \\
\text { ion } \\
\text { Model }\end{array}$ & $\begin{array}{l}\text { kind } \\
\text { of } \\
\text { vario } \\
\text { us } \\
\text { medi } \\
\text { a } \\
\text { prom } \\
\text { otion } \\
\text { s }\end{array}$ & $\begin{array}{l}\text { if not } \\
\text { using a } \\
\text { promot } \\
\text { ional } \\
\text { media }\end{array}$ & $\begin{array}{l}\text { If } \\
\text { using } \\
\text { one } \\
\text { type of } \\
\text { promot } \\
\text { ional } \\
\text { media }\end{array}$ & $\begin{array}{l}\text { If } \\
\text { using } \\
\text { more } \\
\text { than } \\
\text { one } \\
\text { medi } \\
\text { a } \\
\text { camp } \\
\text { aign }\end{array}$ & $\begin{array}{l}\text { If } \\
\text { using } \\
\text { of } \\
\text { various } \\
\text { promot } \\
\text { ional } \\
\text { model } \\
\text { for all } \\
\text { busines } \\
\text { sman } \\
\text { has } \\
\text { been }\end{array}$ & $\begin{array}{l}\text { If using } \\
\text { varietie } \\
\text { s of } \\
\text { media } \\
\text { promot } \\
\text { ions } \\
\text { and } \\
\text { take } \\
\text { advant } \\
\text { age of } \\
\text { coordin } \\
\text { ated }\end{array}$ \\
\hline
\end{tabular}




\begin{tabular}{|c|c|c|c|c|c|c|}
\hline & & & & & $\begin{array}{l}\text { coordin } \\
\text { ated }\end{array}$ & $\begin{array}{l}\text { commu } \\
\text { nicatio } \\
\text { ns } \\
\text { networ } \\
\text { k }\end{array}$ \\
\hline $\begin{array}{l}\text { C. } \\
\text { tourism } \\
\text { inform } \\
\text { ation } \\
\text { system }\end{array}$ & $\begin{array}{l}\text { The } \\
\text { com } \\
\text { binat } \\
\text { ion } \\
\text { of } \\
\text { infor } \\
\text { mati } \\
\text { on } \\
\text { techn } \\
\text { olog } \\
\text { y and } \\
\text { the } \\
\text { activ } \\
\text { ities } \\
\text { of } \\
\text { peop } \\
\text { le } \\
\text { who } \\
\text { use } \\
\text { the } \\
\text { techn } \\
\text { olog } \\
\text { y to } \\
\text { supp } \\
\text { ort } \\
\text { the } \\
\text { activ } \\
\text { ities } \\
\text { of } \\
\text { touri } \\
\text { sm }\end{array}$ & $\begin{array}{l}\text { If not } \\
\text { using } \\
\text { the } \\
\text { inform } \\
\text { ation } \\
\text { system }\end{array}$ & $\begin{array}{l}\text { If using } \\
\text { one } \\
\text { kind of } \\
\text { inform } \\
\text { ation } \\
\text { system } \\
\text { s by } \\
\text { local } \\
\text { tourism } \\
\text { players }\end{array}$ & $\begin{array}{l}\text { If } \\
\text { using } \\
\text { a } \\
\text { wide } \\
\text { varie } \\
\text { ty of } \\
\text { infor } \\
\text { mati } \\
\text { on } \\
\text { syste } \\
\text { ms, } \\
\text { but } \\
\text { is } \\
\text { drive } \\
\text { n by } \\
\text { outsi } \\
\text { ders }\end{array}$ & $\begin{array}{l}\text { If } \\
\text { using } \\
\text { various } \\
\text { forms } \\
\text { system } \\
\text { travel } \\
\text { inform } \\
\text { ation } \\
\text { and } \\
\text { only } \\
\text { driven } \\
\text { by } \\
\text { local } \\
\text { tourism } \\
\text { players }\end{array}$ & $\begin{array}{l}\text { If using } \\
\text { various } \\
\text { forms } \\
\text { of } \\
\text { inform } \\
\text { ation } \\
\text { system } \\
\text { s and } \\
\text { driven } \\
\text { indepe } \\
\text { ndently } \\
\text { by } \\
\text { local } \\
\text { tourism } \\
\text { stakeho } \\
\text { lders } \\
\text { and } \\
\text { outside } \\
\text { stakeho } \\
\text { lder }\end{array}$ \\
\hline $\begin{array}{l}\text { D. } \\
\text { Distrib } \\
\text { ution }\end{array}$ & $\begin{array}{l}\text { prod } \\
\text { ucts } \\
\text { reach } \\
\text { ing } \\
\text { and } \\
\text { relati } \\
\text { onshi } \\
\text { p }\end{array}$ & $\begin{array}{l}\text { If the } \\
\text { product } \\
\text { reachin } \\
\mathrm{g} \& \\
\text { relation } \\
\text { ship is } \\
\text { still } \\
\text { very } \\
\text { local } \\
\text { (one } \\
\text { district } \\
\text { and } \\
\text { surroun } \\
\text { ding } \\
\text { areas } \\
\text { only) }\end{array}$ & $\begin{array}{l}\text { If the } \\
\text { product } \\
\text { reachin } \\
\mathrm{g} \& \\
\text { relation } \\
\text { ship } \\
\text { levels } \\
\text { is } \\
\text { betwee } \\
\mathrm{n} \\
\text { district } \\
\text { s }\end{array}$ & $\begin{array}{l}\text { If } \\
\text { the } \\
\text { prod } \\
\text { uct } \\
\text { reach } \\
\text { ing } \\
\& \\
\text { relati } \\
\text { onshi } \\
\text { p } \\
\text { level } \\
\text { s is } \\
\text { other } \\
\text { provi } \\
\text { nces } \\
\text { in } \\
\text { the } \\
\text { islan } \\
d\end{array}$ & $\begin{array}{l}\text { If the } \\
\text { product } \\
\text { reachin } \\
\mathrm{g} \& \\
\text { relation } \\
\text { ship } \\
\text { levels } \\
\text { is } \\
\text { nationa } \\
1\end{array}$ & $\begin{array}{l}\text { If the } \\
\text { product } \\
\text { reachin } \\
\mathrm{g} \& \\
\text { relation } \\
\text { ship } \\
\text { levels } \\
\text { is } \\
\text { internat } \\
\text { ional } \\
\text { level }\end{array}$ \\
\hline
\end{tabular}

\begin{tabular}{|c|c|c|c|c|c|c|}
\hline \multicolumn{7}{|c|}{ 2. SUSTAINABILITY } \\
\hline \multirow{2}{*}{$\begin{array}{l}\text { PARA } \\
\text { METE } \\
\mathbf{R}\end{array}$} & \multirow{2}{*}{$\begin{array}{l}\text { DET } \\
\text { AIL }\end{array}$} & \multicolumn{5}{|c|}{ VALUES \& INDICATORS } \\
\hline & & $\begin{array}{c}1 \\
\text { point }\end{array}$ & $\begin{array}{l}2 \\
\text { point } \\
\text { s } \\
\end{array}$ & $\begin{array}{l}3 \\
\text { point } \\
\text { s } \\
\end{array}$ & $\begin{array}{l}4 \\
\text { point } \\
\text { s } \\
\end{array}$ & $\begin{array}{l}5 \\
\text { point } \\
\text { s } \\
\end{array}$ \\
\hline $\begin{array}{l}\text { A. } \\
\text { Physic } \\
\text { al } \\
\text { develo } \\
\text { pment } \\
\text { Thresh } \\
\text { olds }\end{array}$ & $\begin{array}{l}\text { The } \\
\text { exten } \\
\text { sive } \\
\text { use of } \\
\text { open } \\
\text { land } \\
\text { for } \\
\text { physi } \\
\text { cal } \\
\text { devel } \\
\text { opme } \\
\text { nt }\end{array}$ & $\begin{array}{l}\text { If } \\
\text { there } \\
\text { is } \\
\text { still } \\
\text { no } \\
\text { regul } \\
\text { ation } \\
\text { of } \\
\text { land } \\
\text { use }\end{array}$ & $\begin{array}{l}\text { If the } \\
\text { existi } \\
\text { ng } \\
\text { arran } \\
\text { geme } \\
\text { nt of } \\
\text { land } \\
\text { use } \\
\text { not } \\
\text { obeye } \\
\text { d }\end{array}$ & $\begin{array}{l}\text { If the } \\
\text { existi } \\
\text { ng } \\
\text { arrang } \\
\text { ement } \\
\mathrm{s} \text { and } \\
\text { restric } \\
\text { tions } \\
\text { on } \\
\text { physi } \\
\text { cal } \\
\text { devel } \\
\text { opme } \\
\text { nt } \\
\text { began } \\
\text { to be } \\
\text { obeye } \\
\text { d }\end{array}$ & $\begin{array}{l}\text { If the } \\
\text { existi } \\
\text { ng } \\
\text { land } \\
\text { use } \\
\text { regula } \\
\text { tion is } \\
\text { obeye } \\
\text { d and } \\
\text { trendi } \\
\text { ly } \\
\text { expan } \\
\text { d } \\
\text { open } \\
\text { space } \\
\text { rather } \\
\text { than } \\
\text { physi } \\
\text { cal } \\
\text { devel } \\
\text { opme } \\
\text { nt }\end{array}$ & $\begin{array}{l}\text { If the } \\
\text { existi } \\
\text { ng } \\
\text { land } \\
\text { use } \\
\text { regul } \\
\text { ation } \\
\text { is } \\
\text { obeye } \\
\text { d and } \\
\text { willin } \\
\text { gness } \\
\text { revisi } \\
\text { on for } \\
\text { physi } \\
\text { cal } \\
\text { devel } \\
\text { opme } \\
\text { nt }\end{array}$ \\
\hline $\begin{array}{l}\text { B. } \\
\text { number } \\
\text { of } \\
\text { visitors } \\
\text { thresho } \\
\text { ld }\end{array}$ & $\begin{array}{l}\text { Num } \\
\text { ber of } \\
\text { visito } \\
\text { rs to } \\
\text { the } \\
\text { vario } \\
\text { us } \\
\text { attrac } \\
\text { tions } \\
\text { of the } \\
\text { carryi } \\
\text { ng } \\
\text { capac } \\
\text { ity } \\
\text { accor } \\
\text { ding } \\
\text { to the } \\
\text { UNW } \\
\text { TO } \\
\text { stand } \\
\text { ard }\end{array}$ & $\begin{array}{l}\text { If } \\
\text { there } \\
\text { is } \\
\text { still } \\
\text { no } \\
\text { path } \\
\text { scena } \\
\text { rio } \\
\text { and } \\
\text { visito } \\
\text { rs } \\
\text { only } \\
\text { unde } \\
\text { rstan } \\
\text { d a } \\
\text { parti } \\
\text { cular } \\
\text { point } \\
\text { of } \\
\text { attrac } \\
\text { tion }\end{array}$ & $\begin{array}{l}\text { If } \\
\text { there } \\
\text { is still } \\
\text { no } \\
\text { tread } \\
\text { path } \\
\text { scena } \\
\text { rio } \\
\text { for } \\
\text { the } \\
\text { flow } \\
\text { of } \\
\text { visito } \\
\text { rs and } \\
\text { the } \\
\text { amou } \\
\text { nt of } \\
\text { build } \\
\text { up } \\
\text { only } \\
\text { at } \\
\text { certai } \\
n \\
\text { points }\end{array}$ & $\begin{array}{l}\text { If } \\
\text { there } \\
\text { is } \\
\text { existi } \\
\text { ng } \\
\text { thread } \\
\text { path } \\
\text { for a } \\
\text { variet } \\
\text { y } \\
\text { point } \\
\text { of } \\
\text { intere } \\
\text { st but } \\
\text { there } \\
\text { is no } \\
\text { mech } \\
\text { anism } \\
\text { for } \\
\text { distrib } \\
\text { uting } \\
\text { the } \\
\text { numb } \\
\text { er of } \\
\text { visitor } \\
\text { s }\end{array}$ & $\begin{array}{l}\text { If } \\
\text { there } \\
\text { is } \\
\text { existi } \\
\text { ng } \\
\text { thread } \\
\text { path } \\
\text { for a } \\
\text { variet } \\
y \text { of } \\
\text { point } \\
\text { of } \\
\text { attract } \\
\text { ion } \\
\text { and a } \\
\text { mech } \\
\text { anism } \\
\text { for } \\
\text { distrib } \\
\text { uting } \\
\text { the } \\
\text { numb } \\
\text { er of } \\
\text { visitor } \\
\mathrm{s}\end{array}$ & $\begin{array}{l}\text { If } \\
\text { existi } \\
\text { ng } \\
\text { threa } \\
\text { d } \\
\text { path } \\
\text { for a } \\
\text { variet } \\
\text { y of } \\
\text { point } \\
\text { of } \\
\text { attrac } \\
\text { tion } \\
\text { and } \\
\text { the } \\
\text { numb } \\
\text { er of } \\
\text { visito } \\
\text { rs } \\
\text { disper } \\
\text { sed as } \\
\text { the } \\
\text { carryi } \\
\text { ng } \\
\text { capac } \\
\text { ity of } \\
\text { each } \\
\text { object }\end{array}$ \\
\hline $\begin{array}{l}\text { C. } \\
\text { natural } \\
\text { resourc } \\
\text { es } \\
\text { Thresh } \\
\text { olds }\end{array}$ & $\begin{array}{l}\text { Suppl } \\
\text { y } \\
\text { capab } \\
\text { ility } \\
\text { and } \\
\text { durab } \\
\text { ility } \\
\text { of } \\
\text { natur } \\
\text { al } \\
\text { resou } \\
\text { rces } \\
\text { on } \\
\text { the } \\
\text { needs } \\
\text { of } \\
\text { touris } \\
\text { ts in }\end{array}$ & $\begin{array}{l}\text { If } \\
\text { there } \\
\text { is } \\
\text { still } \\
\text { no } \\
\text { regul } \\
\text { ation } \\
\text { on } \\
\text { the } \\
\text { use } \\
\text { of } \\
\text { local } \\
\text { natur } \\
\text { al } \\
\text { resou } \\
\text { rces }\end{array}$ & $\begin{array}{l}\text { If } \\
\text { there } \\
\text { is } \\
\text { alread } \\
y \\
\text { regul } \\
\text { ation } \\
\text { on } \\
\text { the } \\
\text { use of } \\
\text { local } \\
\text { natur } \\
\text { al } \\
\text { resou } \\
\text { rces } \\
\text { but } \\
\text { have } \\
\text { not }\end{array}$ & $\begin{array}{l}\text { If the } \\
\text { existi } \\
\text { ng } \\
\text { arrang } \\
\text { ement } \\
\mathrm{s} \text { and } \\
\text { restric } \\
\text { tions } \\
\text { on } \\
\text { natura } \\
1 \\
\text { resour } \\
\text { ces } \\
\text { began } \\
\text { to be } \\
\text { obeye } \\
\text { d }\end{array}$ & $\begin{array}{l}\text { If the } \\
\text { existi } \\
\text { ng } \\
\text { regula } \\
\text { tion } \\
\text { on the } \\
\text { use of } \\
\text { natura } \\
1 \\
\text { resour } \\
\text { ces } \\
\text { tende } \\
\text { ncy to } \\
\text { renew } \\
\text { the } \\
\text { carryi } \\
\text { ng } \\
\text { capaci }\end{array}$ & $\begin{array}{l}\text { If the } \\
\text { existi } \\
\text { ng } \\
\text { regul } \\
\text { ation } \\
\text { on } \\
\text { the } \\
\text { use of } \\
\text { natur } \\
\text { al } \\
\text { resou } \\
\text { rces } \\
\text { obeye } \\
\text { d and } \\
\text { willin } \\
\text { gness } \\
\text { to } \\
\text { contri }\end{array}$ \\
\hline
\end{tabular}




\begin{tabular}{|c|c|c|c|c|c|c|}
\hline & $\begin{array}{l}\text { vario } \\
\text { us } \\
\text { touris } \\
\mathrm{m} \\
\text { object } \\
\mathrm{s}\end{array}$ & & $\begin{array}{l}\text { obeye } \\
\text { d }\end{array}$ & & $\begin{array}{l}\text { ty } \\
\text { resour } \\
\text { ces }\end{array}$ & $\begin{array}{l}\text { bute } \\
\text { to the } \\
\text { renew } \\
\text { al } \\
\text { functi } \\
\text { on of } \\
\text { resou } \\
\text { rces }\end{array}$ \\
\hline $\begin{array}{l}\text { D. } \\
\text { local } \\
\text { commu } \\
\text { nities' } \\
\text { respons } \\
\text { e }\end{array}$ & $\begin{array}{l}\text { React } \\
\text { ion } \\
\text { and } \\
\text { action } \\
\text { of } \\
\text { local } \\
\text { com } \\
\text { munit } \\
\text { y on } \\
\text { touris } \\
\text { m } \\
\text { activi } \\
\text { ties in } \\
\text { the } \\
\text { regio } \\
\mathrm{n}\end{array}$ & $\begin{array}{l}\text { If the } \\
\text { majo } \\
\text { rity } \\
\text { of } \\
\text { local } \\
\text { peopl } \\
\text { e do } \\
\text { not } \\
\text { acce } \\
\text { pt } \\
\text { touri } \\
\text { sm } \\
\text { activi } \\
\text { ties } \\
\text { in the } \\
\text { area }\end{array}$ & $\begin{array}{l}\text { If the } \\
\text { major } \\
\text { ity of } \\
\text { local } \\
\text { peopl } \\
\text { e feel } \\
\text { no } \\
\text { need } \\
\text { to } \\
\text { engag } \\
\text { e the } \\
\text { activi } \\
\text { ties } \\
\text { of } \\
\text { touris } \\
\text { m }\end{array}$ & $\begin{array}{l}\text { If the } \\
\text { major } \\
\text { ity of } \\
\text { local } \\
\text { peopl } \\
\text { e } \\
\text { want } \\
\text { to be } \\
\text { involv } \\
\text { ed in } \\
\text { touris } \\
\text { m } \\
\text { activit } \\
\text { ies }\end{array}$ & $\begin{array}{l}\text { If } \\
\text { comm } \\
\text { unitie } \\
\text { s have } \\
\text { forme } \\
\mathrm{d} \\
\text { organi } \\
\text { zation } \\
\mathrm{s} \text { to } \\
\text { regula } \\
\text { te the } \\
\text { role } \\
\text { of the } \\
\text { touris } \\
\mathrm{m} \\
\text { activit } \\
\mathrm{y}\end{array}$ & $\begin{array}{l}\text { When } \\
\text { the } \\
\text { organ } \\
\text { izatio } \\
\mathrm{n} \\
\text { forme } \\
\mathrm{d} \text { by } \\
\text { com } \\
\text { munit } \\
\text { y } \\
\text { touris } \\
\mathrm{m} \text { has } \\
\text { been } \\
\text { conne } \\
\text { cted } \\
\text { with } \\
\text { vario } \\
\text { us } \\
\text { stake } \\
\text { holde } \\
\text { rs }\end{array}$ \\
\hline $\begin{array}{l}\text { E. } \\
\text { Tourist } \\
\text { 's } \\
\text { Respon } \\
\text { se }\end{array}$ & $\begin{array}{l}\text { React } \\
\text { ion } \\
\text { and } \\
\text { action } \\
\text { touris } \\
\text { ts } \\
\text { who } \\
\text { visit }\end{array}$ & $\begin{array}{l}\text { If } \\
\text { vario } \\
\text { us } \\
\text { attrac } \\
\text { tions } \\
\text { deser } \\
\text { ted } \\
\text { and } \\
\text { stalle } \\
\text { d }\end{array}$ & $\begin{array}{l}\text { If } \\
\text { visite } \\
\mathrm{d} \text { by } \\
\text { indivi } \\
\text { dual } \\
\text { travel } \\
\text { ers } \\
\text { but } \\
\text { no } \\
\text { touris } \\
\mathrm{t} \\
\text { group } \\
\mathrm{s}\end{array}$ & $\begin{array}{l}\text { If the } \\
\text { group } \\
\text { of } \\
\text { tourist } \\
\text { began } \\
\text { to } \\
\text { visit } \\
\text { at } \\
\text { least } \\
\text { by } \\
\text { famil } \\
\text { y } \\
\text { group }\end{array}$ & $\begin{array}{l}\text { If } \\
\text { alread } \\
y \\
\text { marke } \\
\text { ted by } \\
\text { touris } \\
\text { m } \\
\text { busin } \\
\text { esses } \\
\text { corpo } \\
\text { ration }\end{array}$ & $\begin{array}{l}\text { If } \\
\text { start } \\
\text { to be } \\
\text { a list } \\
\text { of } \\
\text { must } \\
\text { visit } \\
\text { for } \\
\text { touris } \\
\text { ts } \\
\text { who } \\
\text { come } \\
\text { to } \\
\text { Bantu } \\
1\end{array}$ \\
\hline $\begin{array}{l}\text { F. } \\
\text { Govern } \\
\text { ment's } \\
\text { respons } \\
\text { e }\end{array}$ & $\begin{array}{l}\text { React } \\
\text { ion } \\
\text { and } \\
\text { action } \\
\text { of } \\
\text { gover } \\
\text { nmen } \\
\mathrm{t} \text { in } \\
\text { the } \\
\text { devel } \\
\text { opme } \\
\text { nt } \\
\text { area }\end{array}$ & $\begin{array}{l}\text { If } \\
\text { there } \\
\text { is no } \\
\text { respo } \\
\text { nse } \\
\text { from } \\
\text { gove } \\
\text { rnme } \\
\text { nt in } \\
\text { plann } \\
\text { ing } \\
\text { the } \\
\text { regio } \\
n\end{array}$ & $\begin{array}{l}\text { If it } \\
\text { listed } \\
\text { in the } \\
\text { regio } \\
\text { nal } \\
\text { devel } \\
\text { opme } \\
\text { nt } \\
\text { agend } \\
\text { a }\end{array}$ & $\begin{array}{l}\text { If it } \\
\text { began } \\
\text { to be } \\
\text { includ } \\
\text { ed in } \\
\text { local } \\
\text { gover } \\
\text { nment } \\
\text { devel } \\
\text { opme } \\
\text { nt } \\
\text { agend } \\
\text { a and } \\
\text { budge } \\
\mathrm{t} \\
\text { alloca } \\
\text { tions }\end{array}$ & $\begin{array}{l}\text { If it } \\
\text { involv } \\
\text { es a } \\
\text { wide } \\
\text { range } \\
\text { of } \\
\text { stakeh } \\
\text { olders } \\
\text { in the } \\
\text { devel } \\
\text { opme } \\
\text { nt of } \\
\text { the } \\
\text { region }\end{array}$ & $\begin{array}{l}\text { If the } \\
\text { provi } \\
\text { ncial } \\
\text { or } \\
\text { natio } \\
\text { nal } \\
\text { gover } \\
\text { nmen } \\
\mathrm{t} \\
\text { maki } \\
\text { ng } \\
\text { plans } \\
\text { and } \\
\text { budge } \\
\mathrm{t} \\
\text { alloca } \\
\text { tions }\end{array}$ \\
\hline
\end{tabular}

\begin{tabular}{|c|c|c|c|c|c|c|}
\hline \multicolumn{7}{|c|}{ 3. PARTICIPATORY } \\
\hline \multirow{2}{*}{$\begin{array}{l}\text { PARA } \\
\text { MET } \\
\text { ER }\end{array}$} & \multirow{2}{*}{$\begin{array}{l}\text { DET } \\
\text { AIL }\end{array}$} & \multicolumn{5}{|c|}{ VALUES \& INDICATORS } \\
\hline & & $\begin{array}{c}1 \\
\text { point }\end{array}$ & $\begin{array}{l} \\
\text { point } \\
\text { S }\end{array}$ & $\begin{array}{l}3 \\
\text { point } \\
\text { S }\end{array}$ & $\begin{array}{l}4 \\
\text { point } \\
\text { S }\end{array}$ & $\begin{array}{l}5 \\
\text { point } \\
\text { S }\end{array}$ \\
\hline $\begin{array}{l}\text { A. } \\
\text { local } \\
\text { resour } \\
\text { ces }\end{array}$ & $\begin{array}{l}\text { The } \\
\text { use of } \\
\text { vario } \\
\text { us } \\
\text { resou } \\
\text { rces } \\
\text { and } \\
\text { local } \\
\text { mater } \\
\text { ials } \\
\text { insigh } \\
\text { ts }\end{array}$ & $\begin{array}{l}\text { If } \\
\text { local } \\
\text { peopl } \\
\text { e do } \\
\text { not } \\
\text { want } \\
\text { to } \\
\text { use } \\
\text { local } \\
\text { mater } \\
\text { ials }\end{array}$ & $\begin{array}{l}\text { If } \\
\text { local } \\
\text { peopl } \\
\text { e } \\
\text { using } \\
\text { local } \\
\text { resou } \\
\text { rces } \\
\text { as } \\
\text { comp } \\
\text { ulsion }\end{array}$ & $\begin{array}{l}\text { If } \\
\text { Local } \\
\text { Peopl } \\
\mathrm{e} \\
\text { want } \\
\text { to use } \\
\text { any } \\
\text { local } \\
\text { resou } \\
\text { rces } \\
\text { as a } \\
\text { suppl } \\
\text { ement }\end{array}$ & $\begin{array}{l}\text { If the } \\
\text { major } \\
\text { ity of } \\
\text { local } \\
\text { peopl } \\
\text { e } \\
\text { using } \\
\text { local } \\
\text { resour } \\
\text { ces }\end{array}$ & $\begin{array}{l}\text { If the } \\
\text { local } \\
\text { peopl } \\
\mathrm{e} \\
\text { maxi } \\
\text { mizin } \\
\mathrm{g} \text { in } \\
\text { using } \\
\text { local } \\
\text { resour } \\
\text { ces } \\
\text { and } \\
\text { make } \\
\text { it as a } \\
\text { theme } \\
\text { of } \\
\text { touris } \\
\mathrm{m} \\
\text { area }\end{array}$ \\
\hline $\begin{array}{l}\text { B. } \\
\text { local } \\
\text { accoun } \\
\text { tability }\end{array}$ & $\begin{array}{l}\text { The } \\
\text { invol } \\
\text { veme } \\
\text { nt of } \\
\text { local } \\
\text { com } \\
\text { munit } \\
\text { ies } \\
\text { for } \\
\text { the } \\
\text { mana } \\
\text { geme } \\
\text { nt } \\
\text { and } \\
\text { devel } \\
\text { opme } \\
\text { nt of } \\
\text { organ } \\
\text { izatio } \\
\text { ns }\end{array}$ & $\begin{array}{l}\text { If } \\
\text { local } \\
\text { peopl } \\
\text { e do } \\
\text { not } \\
\text { want } \\
\text { to get } \\
\text { invol } \\
\text { ved } \\
\text { in the } \\
\text { activi } \\
\text { ties } \\
\text { of } \\
\text { touris } \\
\text { m }\end{array}$ & $\begin{array}{l}\text { If } \\
\text { local } \\
\text { peopl } \\
\text { e } \\
\text { want } \\
\text { to be } \\
\text { invol } \\
\text { ved } \\
\text { in } \\
\text { touris } \\
\text { m } \\
\text { activi } \\
\text { ty but } \\
\text { do } \\
\text { not } \\
\text { want } \\
\text { to } \\
\text { form } \\
\text { organ } \\
\text { izatio } \\
\text { ns }\end{array}$ & $\begin{array}{l}\text { If } \\
\text { there } \\
\text { are } \\
\text { touris } \\
\mathrm{m} \\
\text { organ } \\
\text { izatio } \\
\text { ns but } \\
\text { there } \\
\text { is no } \\
\text { activi } \\
\text { ty } \\
\text { activi } \\
\text { ty }\end{array}$ & $\begin{array}{l}\text { If } \\
\text { touris } \\
\mathrm{m} \\
\text { organi } \\
\text { zation } \\
\text { start } \\
\text { beco } \\
\text { me as } \\
\text { a } \\
\text { regula } \\
\text { tor of } \\
\text { local } \\
\text { comm } \\
\text { unity } \\
\text { activit } \\
\text { ies }\end{array}$ & $\begin{array}{l}\text { If the } \\
\text { organi } \\
\text { zation } \\
\text { has } \\
\text { been } \\
\text { associ } \\
\text { ated } \\
\text { with } \\
\text { cross- } \\
\text { sector } \\
\text { al } \\
\text { stakeh } \\
\text { olders }\end{array}$ \\
\hline $\begin{array}{l}\mathrm{C} . \\
\text { local } \\
\text { variety }\end{array}$ & $\begin{array}{l}\text { The } \\
\text { uniqu } \\
\text { eness } \\
\text { of the } \\
\text { vario } \\
\text { us } \\
\text { local } \\
\text { touris } \\
\text { t } \\
\text { attrac } \\
\text { tion }\end{array}$ & $\begin{array}{l}\text { If } \\
\text { there } \\
\text { is not } \\
\text { uniqu } \\
\text { eness } \\
\text { of the } \\
\text { area }\end{array}$ & $\begin{array}{l}\text { If } \\
\text { there } \\
\text { is } \\
\text { alread } \\
y \\
\text { uniqu } \\
\text { eness } \\
\text { of the } \\
\text { area } \\
\text { but } \\
\text { not } \\
\text { been } \\
\text { packe } \\
\text { d yet }\end{array}$ & $\begin{array}{l}\text { If } \\
\text { some } \\
\text { existi } \\
\text { ng } \\
\text { local } \\
\text { uniqu } \\
\text { eness } \\
\text { been } \\
\text { packa } \\
\text { ged } \\
\text { for } \\
\text { touris } \\
\text { m } \\
\text { produ } \\
\text { cts }\end{array}$ & $\begin{array}{l}\text { If the } \\
\text { local } \\
\text { uniqu } \\
\text { eness } \\
\text { has } \\
\text { beco } \\
\text { me } \\
\text { the } \\
\text { collec } \\
\text { tive } \\
\text { consci } \\
\text { ousne } \\
\text { ss as } \\
\text { touris } \\
\text { m } \\
\text { produ } \\
\text { ct }\end{array}$ & $\begin{array}{l}\text { If the } \\
\text { local } \\
\text { uniqu } \\
\text { eness } \\
\text { has } \\
\text { beco } \\
\text { me } \\
\text { the } \\
\text { collec } \\
\text { tive } \\
\text { consci } \\
\text { ousne } \\
\text { ss and } \\
\text { packe } \\
\text { d and } \\
\text { protec } \\
\text { ted by } \\
\text { legal } \\
\text { instru } \\
\text { ments }\end{array}$ \\
\hline $\begin{array}{l}\text { D. The } \\
\text { econo } \\
\text { my } \\
\text { advant } \\
\text { age for } \\
\text { local } \\
\text { people }\end{array}$ & $\begin{array}{l}\text { Distri } \\
\text { butio } \\
\mathrm{n} \text { of } \\
\text { inco } \\
\text { me } \\
\text { receiv } \\
\text { ed by } \\
\text { the }\end{array}$ & $\begin{array}{l}\text { If } \\
\text { there } \\
\text { is no } \\
\text { econ } \\
\text { omic } \\
\text { benef } \\
\text { it } \\
\text { recei }\end{array}$ & $\begin{array}{l}\text { If } \\
\text { there } \\
\text { is } \\
\text { alread } \\
\text { y a } \\
\text { small } \\
\text { part } \\
\text { of }\end{array}$ & $\begin{array}{l}\text { If } \\
\text { most } \\
\text { of the } \\
\text { travel } \\
\text { needs } \\
\text { can } \\
\text { be } \\
\text { suppli }\end{array}$ & $\begin{array}{l}\text { If an } \\
\text { existi } \\
\text { ng } \\
\text { busin } \\
\text { ess } \\
\text { entity/ } \\
\text { organi } \\
\text { zation }\end{array}$ & $\begin{array}{l}\text { If the } \\
\text { busin } \\
\text { ess } \\
\text { institu } \\
\text { tion } \\
\text { forme } \\
\text { d by } \\
\text { local }\end{array}$ \\
\hline
\end{tabular}




\begin{tabular}{|l|l|l|l|l|l|l|} 
local & ved & com & ed by & to & peopl \\
com & by & munit & a & distrib & e has \\
munit & local & y & variet & ute & associ \\
y and & com & mem & y of & most & ated \\
direct & munit & bers & backg & profit & with \\
busin & ies & receiv & round & for & the \\
ess & & e & s of & local & major \\
playe & & econo & indivi & peopl & ity of \\
r & & mic & duals & e & citize \\
benef & in & & ns and \\
extern \\
\end{tabular}

\begin{tabular}{|c|c|c|c|c|c|c|}
\hline \multicolumn{7}{|c|}{ 4. DISASTER MITIGATION } \\
\hline \multirow{2}{*}{$\begin{array}{l}\text { PARA } \\
\text { METE } \\
\text { R }\end{array}$} & \multirow{2}{*}{$\begin{array}{l}\text { DET } \\
\text { AIL }\end{array}$} & \multicolumn{5}{|c|}{ VALUES \& INDICATORS } \\
\hline & & $\begin{array}{c}1 \\
\text { point }\end{array}$ & $\begin{array}{l}2 \\
\text { points }\end{array}$ & $\begin{array}{l}3 \\
\text { point } \\
\text { S }\end{array}$ & $\begin{array}{l}4 \\
\text { poin } \\
\text { ts }\end{array}$ & $\begin{array}{l}5 \\
\text { poin } \\
\text { ts }\end{array}$ \\
\hline $\begin{array}{l}\text { A. The } \\
\text { earthq } \\
\text { uake } \\
\text { and } \\
\text { landsli } \\
\text { des }\end{array}$ & $\begin{array}{l}\text { Anti } \\
\text { cipat } \\
\text { ion } \\
\text { of } \\
\text { risk } \\
\text { and } \\
\text { reco } \\
\text { very } \\
\text { plan } \\
\text { ning }\end{array}$ & $\begin{array}{l}\text { If there } \\
\text { are no } \\
\text { anticip } \\
\text { ation } \\
\text { and } \\
\text { rescue } \\
\text { plans } \\
\text { from } \\
\text { earthqu } \\
\text { akes } \\
\text { and or } \\
\text { landsli } \\
\text { des }\end{array}$ & $\begin{array}{l}\text { If there } \\
\text { are } \\
\text { anticip } \\
\text { ation } \\
\text { and a } \\
\text { rescue } \\
\text { plan } \\
\text { for } \\
\text { earthqu } \\
\text { akes } \\
\text { and } \\
\text { landsli } \\
\text { des but } \\
\text { has not } \\
\text { been } \\
\text { socializ } \\
\text { ed }\end{array}$ & $\begin{array}{l}\text { If } \\
\text { there } \\
\text { are } \\
\text { antici } \\
\text { patio } \\
\mathrm{n} \\
\text { plan, } \\
\text { socia } \\
\text { lizati } \\
\text { on } \\
\text { and } \\
\text { rescu } \\
\text { e } \\
\text { traini } \\
\text { ng }\end{array}$ & $\begin{array}{l}\text { If } \\
\text { there } \\
\text { are } \\
\text { evac } \\
\text { uatio } \\
\mathrm{n} \\
\text { plan } \\
\text { and a } \\
\text { speci } \\
\text { al } \\
\text { team } \\
\text { to } \\
\text { hand } \\
\text { le } \\
\text { the } \\
\text { disas } \\
\text { ter } \\
\text { by } \\
\text { the } \\
\text { local } \\
\text { peop } \\
\text { le }\end{array}$ & $\begin{array}{l}\text { If } \\
\text { there } \\
\text { are a } \\
\text { plan, } \\
\text { SAR } \\
\text { Tea } \\
\text { m, } \\
\text { and } \\
\text { the } \\
\text { post- } \\
\text { disas } \\
\text { ter } \\
\text { reco } \\
\text { very } \\
\text { area } \\
\text { plan }\end{array}$ \\
\hline $\begin{array}{l}\text { B. } \\
\text { non- } \\
\text { natural } \\
\text { disaste } \\
\text { rs as } \\
\text { effect } \\
\text { of } \\
\text { failed } \\
\text { technol } \\
\text { ogy, } \\
\text { pestile } \\
\text { nce }\end{array}$ & $\begin{array}{l}\text { Anti } \\
\text { cipat } \\
\text { ion } \\
\text { of } \\
\text { hum } \\
\text { an } \\
\text { error } \\
\text { and } \\
\text { stand } \\
\text { ardiz } \\
\text { e the } \\
\text { safet } \\
\text { y and } \\
\text { healt } \\
\text { h }\end{array}$ & $\begin{array}{l}\text { If there } \\
\text { is no } \\
\text { anticip } \\
\text { ation } \\
\text { and } \\
\text { standar } \\
\text { dizatio } \\
\mathrm{n} \text { for } \\
\text { minim } \\
\text { um } \\
\text { safety } \\
\text { and } \\
\text { health }\end{array}$ & $\begin{array}{l}\text { If } \\
\text { already } \\
\text { have a } \\
\text { standar } \\
\text { dizatio } \\
\text { n but } \\
\text { has not } \\
\text { been } \\
\text { used as } \\
\text { a } \\
\text { referen } \\
\text { ce }\end{array}$ & $\begin{array}{l}\text { If } \\
\text { safet } \\
\text { y \& } \\
\text { healt } \\
\mathrm{h} \\
\text { stand } \\
\text { ard } \\
\text { is } \\
\text { alrea } \\
\text { dy } \\
\text { beco } \\
\text { ming } \\
\text { a } \\
\text { refer } \\
\text { ence } \\
\text { for } \\
\text { local } \\
\text { touris } \\
\mathrm{m} \\
\text { stake } \\
\text { holde } \\
\text { rs } \\
\text { and } \\
\text { touris } \\
\mathrm{t}\end{array}$ & $\begin{array}{l}\text { If } \\
\text { there } \\
\text { is an } \\
\text { inter } \\
\text { natio } \\
\text { nal } \\
\text { stand } \\
\text { ardiz } \\
\text { ation } \\
\text { of } \\
\text { safet } \\
y \\
\text { and } \\
\text { healt } \\
h \text { are } \\
\text { obey } \\
\text { ed }\end{array}$ & $\begin{array}{l}\text { If } \\
\text { there } \\
\text { is } \\
\text { sanct } \\
\text { ions/ } \\
\text { penal } \\
\text { ty } \\
\text { for } \\
\text { disob } \\
\text { eyed } \\
\text { healt } \\
\text { h } \\
\text { and } \\
\text { safet } \\
\text { y } \\
\text { stand } \\
\text { ardiz } \\
\text { ation } \\
\text { by } \\
\text { local } \\
\text { peop } \\
\text { le } \\
\text { and } \\
\text { touri } \\
\text { st }\end{array}$ \\
\hline
\end{tabular}

Source: Inspect.id team, 2016

\section{Conclusion}

MSP+DM analyisis helps ecotourism stakeholder to develop the destination area because quantifications of the analysis process during planning and preopening process will help stakeholder and local community easier to understand what will they do. The planning target for stakeholders also can be seen in MSP+DM so the activities in the future can be determined easily.

\section{References}

Ayub, A. et al. (2013) 'A conceptual framework on evaluating SWOT analysis as the mediator in strategic marketing planning through marketing intelligence', European Journal of Business and Social Sciences, 2(1), pp. 91-98.

BAPPEDA (2009) Profil Daerah Provinsi D.I. Yogyakarta. Yogyakarta: Badan Perencanaan Pembangunan.

\section{BPS (2017) . Jakarta: BPS-Statistics} Indonesia.

Dashper, K. (2015) Rural tourism: An international perspective. Cambridge Scholars Publishing.

Dolnicar, S. (2008) 'Market segmentation in tourism', Tourism management, analysis, behaviour and strategy. Cabi Cambridge, MA, pp. 129-150.

Dorobantu, M. R. and Nistoreanu, P. (2012) 'Rural tourism and ecotourism-the main priorities in sustainable development orientations of rural local communities in Romania', Economy Transdisciplinarity Cognition. George Bacovia University, 15(1), p. 259.

Oreski, D. (2012) 'Strategy development by using SWOT-AHP', Tem Journal, 1(4), pp. 283-291. 
Priatmoko, S. (2017a) 'Analysis of Marketability, Sustainability, Participatory and Disaster Mitigation (MSP+DM) for the Development of Rural Community-Based Tourism (CBT) Destinations Case Study: Depok Beach, Bantul, Yogyakarta', in CITIES 2017 International Conference, Multi Perspectives on Peri - Urban Dynamics Towards Sustainable Development. Surabaya, Indonesia: Department of Urban and Regional Planning Institut Teknologi Sepuluh Nopember (ITS), Indonesia.

Priatmoko, S. (2017b) 'Pengaruh Atraksi, Mediasosial, Dan Infrastruktur Terhadap Keputusan Berkunjung Wisatawan Ke Desa Wisata Pentingsari Yogyakarta', Khasanah Ilmu, 8(1), pp. 72-82.

Purbadi, D. (2016) 'Model Pengukuran Kondisi Awal sebagai Dasar Pengembangan Program Pemberdayaan Masyarakat Berkelanjutan, Studi Kasus: Kawasan Wisata Pantai Baru, Kabupaten Bantul', Prosiding Sendimas, (1), pp. 110-121.

Sayyed, M. R. G. (2013) 'SWOT analysis of Tandooreh National Park (NE Iran) for sustainable ecotourism', Proceedings of the International Academy of Ecology and Environmental Sciences. International Academy of Ecology and Environmental Sciences (IAEES), 3(4), p. 296.

Spencer, D. M. (2010) 'Facilitating public participation in tourism planning on American Indian reservations: A case study involving the Nominal Group Technique', Tourism Management. Elsevier, 31(5), pp. 684-690.

Susmayadi, I. M. et al. (2014) 'Sustainable disaster risk reduction through effective risk communication media in Parangtritis tourism area, Yogyakarta', Procedia Environmental Sciences. Elsevier, 20, pp. 684-692. 